\section{Wer mit Benzos kombiniert, riskiert Opioidüberdosis}

\begin{abstract}
Mit der Zunahme von Opioidverschreibungen steigt das Risiko von Überdosierungen. Besonders gefährlich wird es, wenn die opiatartigen Schmerzmittel mit Benzodiazepinen kombiniert werden.
\end{abstract}

W elche Folgen die Kombination von Opioidanalgetika mit Benzodiazepinen zeitigt, haben US-Forscher um Eric Sun von der Stanford University in Kalifornien untersucht. Sie nahmen sich dafür die Krankenversicherungsdaten von mehr als 300.000 Patienten vor, die im Zeitraum von 2001 bis 2013 mindestens ein Opioidrezept eingelöst hatten.

Für Patienten, die Opioide, aber keine Benzodiazepine einnahmen, lag die jährliche Inzidenz für eine durch Opioidüberdosierung bedingte Notfallaufnahme oder stationäre Einweisung bei $1,16 \%$. Wurden zu den Opioiden noch Benzodiazepine verabreicht, stieg die Quote auf 2,42\%. Nach dem Abgleich gegen Alter und eine Reihe von Begleitkrankheiten errechneten Sun und Kollegen ein rund doppelt so hohes Überdosierungsrisiko unter der Kombinationstherapie wie für die Opioidgabe allein.

Erhielten die Patienten die opioidhaltigen Schmerzmedikamente nur intermittierend, ging der Zusatz von Benzo- diazepinen mit einer Erhöhung des Risiko für eine Opioidüberdosis um etwa $40 \%$ einher, von jährlich 1,02\% auf $1,45 \%$. Bei chronischer Opioideinnahme betrug die jährliche Inzidenz 3,13\% ohne und 5,36\% mit Benzodiazepinen, was in der adjustierten Kalkulation einer mit den Tranquilizern assoziierten Erhöhung um rund $80 \%$ entsprach.

„Vorausgesetzt, diese Beziehung ist kausal, würde das Vermeiden von Opioid-Benzodiazepin-Kombinationen die durch eine Opioidüberdosis bedingten Besuche in der Notaufnahme beziehungsweise stationären Einweisungen um 15\% senken“, schreiben Sun und Mitarbeiter. Die gleichzeitige Einnahme dieser Substanzen trage wesentlich zum Populationsrisiko der Überdosierung von Opioiden bei.

Die Studie verdeutlichte zudem, dass während der untersuchten Zeitspanne von 2001 bis 2013 der Anteil von Patienten unter Opioiden, die gleichzeitig Benzodiazepine einnahmen, von $9 \%$ auf
$17 \%$ gestiegen ist. Bei rund $30 \%$ der tödlich verlaufenden Opioidvergiftungen seien Benzodiazepine im Spiel, so die Forscher. Dies werfe die Frage auf, ob die den Opioiden zugeschriebenen Todesfälle nicht teilweise durch die vermehrte Kombination dieser Substanzen mit Benzodiazepinen verursacht worden sein könnten. Benzodiazepine verstärken die atemdepressive Wirkung von Opioiden.

Generell hat sich in den USA im ersten Jahrzehnt des Jahrhunderts die Zahl der verschriebenen Opioide vervierfacht. Auch in Deutschland haben die Opioidverordnungen zugenommen, aber bei Weitem nicht in diesem Ausmaß. Eine Erhebung auf der Basis von Versichertendaten ergab im Jahr 2013 eine Zunahme der Verschreibungen um $37 \%$, und zwar von $3,31 \%$ auf $4,53 \%$ aller Versicherten, für den Zeitraum von 2000 bis 2010.

Fazit: In der Praxis muss die Indikationsstellung für eine Kombination von Benzodiazepinen mit Opioiden besonders eng gefasst werden.

Dr. Robert Bublak

Sun EC et al. Association between concurrent use of prescription opioids and benzodiazepines and overdose: retrospective analysis. BMJ 2017; 356: j760

\section{Karpaltunnelsyndrom: manuelle Therapie ebenso effektiv wie OP}

\begin{abstract}
Beim Karpaltunnelsyndrom ist nicht nur die Operation zielführend, um die Beschwerden des Patienten zu lindern. Mittel- bis langfristig wurden in einer spanischen Studie mit einer multimodalen manuellen Therapie ähnliche Effekte im Hinblick auf Druckschmerzempfindlichkeit und Schmerzintensität erzielt.
\end{abstract}

$\mathrm{M}$ enschen mit Karpaltunnelsyndrom (KTS) leiden unter anderem an ausstrahlenden Schmerzen und Temperaturüberempfindlichkeit infolge einer zentralen Sensibilisierung. Wie sich darauf manuelle Therapien im Vergleich zu operativen Eingriffen auswirken, haben César Fernández de las Peñas von der Universität Rey Juan Carlos in Alcorcón und Kollegen an einer Madrider Klinik untersucht.

In der randomisierten, verblindeten Studie mit 100 Frauen mit KTS erhielten 50 Patientinnen eine manuelle Therapie (wöchentlich, insgesamt drei 30-minütige Behandlungen einschließlich desensibilisierender Maßnahmen) und 50 Frauen wurden chirurgisch versorgt. Bei den Studienteilnehmerinnen bestanden seit mindestens sechs Monaten Schmerzen und Parästhesien in den Aufzweigungen des Nervus medianus; Tinelund Phalen-Test waren positiv. Zudem zeigten sich Beeinträchtigungen der Reizleitung beim Medianusnerv. Ermittelt wurden die Druckschmerzschwelle (PPT), die Temperaturschmerzschwelle (HPT oder CPT) sowie die Schmerzstärke (auf einer Skala von 0-10) zu Beginn der Studie sowie drei, sechs, neun und zwölf Monate nach der Intervention. Von 95 Patientinnen lagen die Daten aus zwölf Monaten vor.

Bei Patientinnen, die eine manuelle Therapie erhalten hatten, ergab sich nach drei, sechs und neun Monaten ein stärkerer Anstieg der Druckschmerzschwelle über dem Karpaltunnel als bei den 